\title{
Wilsonian Effective Actions and the IR/UV Mixing in Noncommutative Gauge Theories
}

\author{
Valentin V. Khoze and Gabriele Travaglini \\ Department of Physics and IPPP, University of Durham, Durham, DH1 3LE, UK \\ E-mail: valya.khoze@durham.ac.uk, gabriele.travaglini@durham.ac.uk
}

\begin{abstract}
Using background field perturbation theory we study Wilsonian effective actions of noncommutative gauge theories with an arbitrary matter content. We determine the Wilsonian coupling constant and the gauge boson polarization tensor as functions of the momentum scale $k$ at the one-loop level and study their short-distance behaviour as $\theta \cdot k \rightarrow 0$, where $\theta$ is the noncommutativity parameter. The mixing between the short-distance and the long-distance degrees of freedom characteristic of noncommutative field theories violates the universality of the Wilsonian action and leads to IR-singularities. We find, in agreement with known results, that the quadratic IR divergences cancel in supersymmetric gauge theories. The logarithmic divergences disappear in mass-deformed $\mathcal{N}=4$ theories, but not in other finite $\mathcal{N}=2$ theories. We next concentrate on finite $\mathcal{N}=2$ and mass-deformed $\mathcal{N}=4$ supersymmetric $U(1)$ gauge theories with massive hypermultiplets. The Wilsonian running coupling exhibits a non-trivial threshold behaviour at and well below the noncommutativity scale $1 / \sqrt{\theta}$, eventually becoming flat in the extreme infrared in $\mathcal{N}=4$ theories, but not in $\mathcal{N}=2$ theories. This is interpreted as the (non)-existence of a non-singular commutative limit where the theory is described by a commutative $\mathcal{N}=2$ pure $U(1)$ theory. We expect that our analysis of finite theories is exact to all orders in perturbation theory.
\end{abstract}




\section{Introduction}

Gauge theories on noncommutative spaces have recently attracted much attention for their applications to string and matrix theories [1-3]. Supersymmetric gauge theories arise as the low-energy description of open strings ending on D-branes in the presence of a constant $B$-field which gives rise to space-noncommutativity,

$$
\left[x^{\mu}, x^{\nu}\right]=i \theta^{\mu \nu}
$$

In this paper we analyze the field theory dynamics of noncommutative gauge theories in four spacetime dimensions and in the presence of a generic matter field content. For related recent work see [4-20] and references therein. Noncommutative field theories can be defined by replacing the ordinary products of fields in the Lagrangians of their commutative counterparts by the star-products

$$
(\phi \star \chi)(x) \equiv \phi(x) e^{\frac{i}{2} \theta^{\mu \nu} \overleftarrow{\partial_{\mu}} \overrightarrow{\partial_{\nu}}} \chi(x)
$$

In this way noncommutative theories can be viewed as field theories on ordinary commutative spacetime. For example, the noncommutative pure gauge theory action is

$$
S_{\mathrm{YM}}[A]=-\frac{1}{2 g^{2}} \int d^{4} x \operatorname{Tr}\left(F_{\mu \nu} \star F_{\mu \nu}\right),
$$

where the field strength is defined as $F_{\mu \nu}=\partial_{\mu} A_{\nu}-\partial_{\nu} A_{\mu}+\left[A_{\mu}, A_{\nu}\right]_{\star}$. If we Taylor-expand the star-products in (1.3) we obtain the action of the standard commutative theory plus an infinite number of higher-derivative terms. At an energy-scale below the noncommutativity scale, $k^{2} \ll 1 / \theta$, the higher-derivative terms correspond to irrelevant operators. One would normally expect that in the infrared one can simply drop all the effects due to irrelevant operators. This would imply that the noncommutative and the corresponding commutative theories belong to the same universality class, i.e. in the infrared their behaviour is identical. Classically the two theories are, in fact, identical in this regime. But it turns out [4,5] that this naive universality is invalidated at the quantum level due to a curious mixing between the short-distance and the long-distance modes in the loop expansion of noncommutative theories.

Standard commutative theories are known to be universal, i.e. their UV-modes are decoupled from the IR-modes. This universality apparently goes wrong in noncommutative theories. The reason for this lies in the UV-properties of these theories. It is believed that the noncommutative theories are UV-renormalizable (when their commutative counterparts are) contrary to the naive expectations about irrelevant higher-derivative operators. In perturbation theory $[5,6]$ the loop integrals in the planar diagrams of the noncommutative theory are exactly the same as in the commutative counterpart. The non-planar diagrams, however, are multiplied by phase factors of the form $e^{i k \cdot \theta \cdot p}$, where $k$ are external momenta, and $p$ are loop momenta. 
These oscillating phases improve the UV-convergence of the non-planar diagrams and typically render them finite. This is basically the reason for the UV-renormalizability of the noncommutative theory ${ }^{1}$. But in the IR limit of the external momenta, $k \rightarrow 0$, the phases vanish and the non-planar diagram diverge, but this divergence is now interpreted as an IR-divergence $[4,5]$. This is the origin of the IR/UV mixing in the noncommutative theories and it leads to the breakdown of universality.

In this paper we study the IR/UV mixing, (non)-universality and the IR commutative limits $\theta k \rightarrow 0$ of generic supersymmetric and non-supersymmetric gauge theories in the Wilsonian approach, which is well suited for addressing the issues of the IR dynamics. We evaluate and discuss the Wilsonian running coupling and the gauge boson polarization tensor as functions of the Wilsonian momentum scale $|k|$ at the one-loop level. The IR/UV mixing violates universality of the Wilsonian action and leads to the IR-singularities: $(\theta \cdot k)^{-2}$ divergences in the polarization tensor and new $\log (k \cdot \theta \cdot k)$ divergences in the coupling in addition to the standard $\theta$-independent running. We find, in agreement with known results [4], that the $(\theta \cdot k)^{-2}$ divergences cancel in all supersymmetric theories. The $\log (k \cdot \theta \cdot k)$ divergences disappear in finite $\mathcal{N}=4$ supersymmetric theories (with or without supersymmetry breaking mass terms) in agreement with [4], but not in finite $\mathcal{N}=2$ theories with fundamental hypermultiplets.

We analyze in detail the running of the Wilsonian coupling in supersymmetric noncommutative $U(1)$ theories with asymptotically free and vanishing microscopic $\beta$ functions. All these theories are not universal, and in the commutative limit their dynamics are different from the naive expectations. Equations (6.4) and (6.7) summarize our results for the running of the Wilsonian coupling constants in the noncommutative finite supersymmetric theories, as shown in Figures 1 and 2 respectively. Let us first describe the results of our analysis of the $\mathcal{N}=4$ theory softly broken to $\mathcal{N}=2$ by the adjoint hypermultiplet mass term. From now on this will be referred to as the $\mathcal{N}=2^{*}$ theory. Figure 1 shows the running of the Wilsonian coupling of this theory in four different regions. The first one is the UV region, $k>>m$, where $m$ is the mass of the adjoint chiral multiplets. Here the coupling is flat as it should be for the $\mathcal{N}=4$ noncommutative theory. In the second region, $\theta^{-1 / 2}<<k<<m$, the coupling is asymptotically free, again in agreement with expectations, since in this regime the massive hypermultiplets are integrated out and the theory is simply a pure noncommutative $\mathcal{N}=2$ $U(1)$ (which is asymptotically free). In the third region, $k<\theta^{-1 / 2}$, the coupling exhibits a non-trivial threshold behaviour at and well below the noncommutativity scale $\theta^{-1 / 2}$, eventually becoming flat in the extreme infrared, $k<<\theta^{-1 / 2}$ (in the region four). This is interpreted as the existence of a non-singular commutative limit where the theory is described by a commutative $\mathcal{N}=2$ pure $U(1)$ theory which indeed has $\beta=0$.

\footnotetext{
${ }^{1}$ Note, that if we had Taylor-expanded the star-products, we would have ended up with the Taylor expansion for the phase factors rendering each term in the expansion progressively more singular in the UV region of loop momenta.
} 


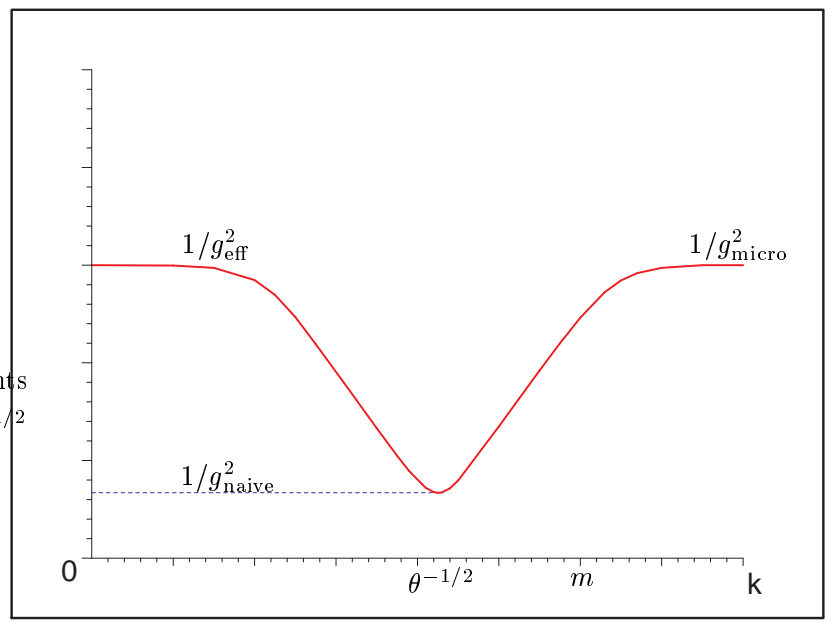

Figure 1. Logarithmic plot of the Wilsonian coupling of the $\mathcal{N}=2^{*}$ theory with mass $m$. The dashed line represents the naive expectation for the low-energy commutative effective theory.

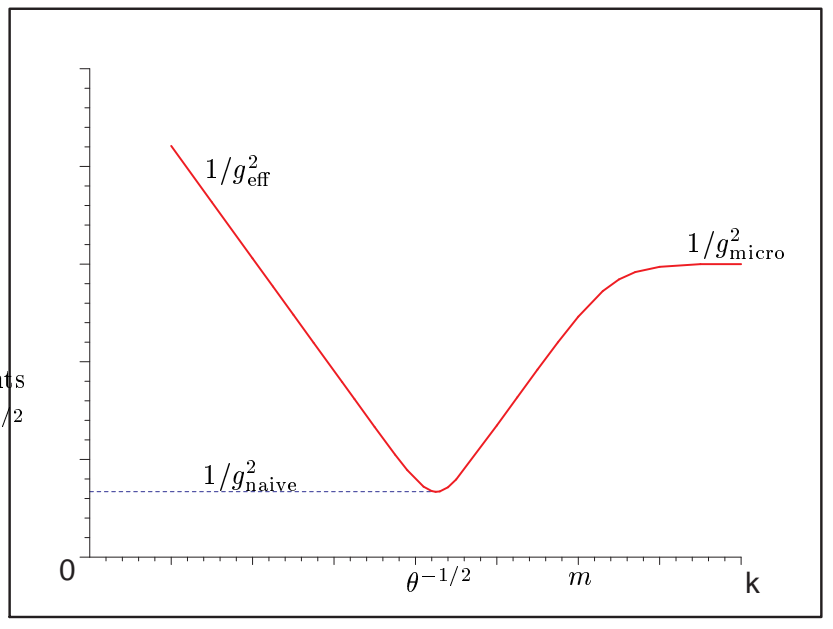

Figure 2. Logarithmic plot of the Wilsonian coupling of the finite $\mathcal{N}=2 U(1)$ theory with $N_{f}=2$ fundamental hypermultiplets of mass $m$. 
The Wilsonian coupling of the noncommutative finite $\mathcal{N}=2 U(1)$ theory with $N_{\mathbf{f}}=2$ fundamental hypermultiplets with mass $m$ as a function of the momentum scale is shown in Figure 2. Here the Wilsonian coupling exhibits the screening behaviour all the way below the noncommutativity scale $1 / \sqrt{\theta}$. This has no resemblance to a commutative $\mathcal{N}=2$ pure $U(1)$ theory, which is supposed to be flat. Somehow, in the IR the theory becomes commutative, but not the same flat commutative one as one would expect in the classical limit. We interpret this fact as a loss of universality in the IR regime.

Figures 1 and 2 show that, remarkably, the effective coupling $g_{\text {eff }}$ never explodes $\left(\frac{1}{g_{\text {eff }}^{2}}\right.$ does not reach zero) for not too large values of the microscopic coupling $g_{\text {micro }}$.

The organization of the rest of the paper is as follows. In Section 2 we introduce the background field method for noncommutative gauge theories which is used for calculating the Wilsonian effective action in perturbation theory. In Section 3 we present the Feynman rules which are used in Sections 4 and 5 to compute the 1-loop determinants arising from integrating out the fluctuating fields. Then the Wilsonian running coupling constant and the gauge boson polarization tensor are computed as functions of the momentum scale $k$. We also discuss the simplifications which occur in supersymmetric theories. In Section 6 we analyze the Wilsonian flow of finite massive supersymmetric gauge theories down to the infrared. To fully exploit our formalism, we compute in Section 7 the evolution of the Wilsonian coupling constant for asymptotically free pure $\mathcal{N}=1$ and $\mathcal{N}=2$ Super Yang-Mills theories. Finally, in the Appendix we present an alternative simple derivation of the microscopic $\beta$ function for noncommutative $U(N)$ gauge theories counting zero modes of noncommutative instantons.

\section{Note on Conventions}

Throughout the paper we work in Euclidean space. Our $\sigma_{\mu}$ and $\bar{\sigma}_{\mu}$ matrices are defined as $\sigma_{\mu}=\left(i \sigma^{m}, \mathbb{1}_{2 \times 2}\right)$ and $\bar{\sigma}_{\mu}=\left(-i \sigma^{m}, \mathbb{1}_{2 \times 2}\right)$ in terms of the three Pauli matrices $\sigma^{m}$. We will also use $\sigma_{\mu \nu}=\frac{1}{2}\left(\sigma_{\mu} \bar{\sigma}_{\nu}-\sigma_{\nu} \bar{\sigma}_{\mu}\right)=i \eta_{\mu \nu}^{a} \sigma^{a}$, and $\bar{\sigma}_{\mu \nu}=\frac{1}{2}\left(\bar{\sigma}_{\mu} \sigma_{\nu}-\bar{\sigma}_{\nu} \sigma_{\mu}\right)=i \bar{\eta}_{\mu \nu}^{a} \sigma^{a}$, where $\eta_{\mu \nu}^{a}$ and $\bar{\eta}_{\mu \nu}^{a}$ are the 't Hooft symbols [24].

For the general discussion of the gauge group $U(N)$ in Section 2 and in the Appendix we use anti-hermitian gauge-group generators $t^{A}$ with the normalization $\operatorname{Tr}\left(t^{A} t^{B}\right)=-\frac{\delta^{A B}}{2}$. Hence the generator of the $U(1)$ component of $U(N)$ is $t^{0}=\frac{1}{i \sqrt{2 N}}$.

In Sections 3-7 we concentrate on the $U(1)$ gauge group and change the normalization of the $U(1)$ generator to $t^{0}=\frac{1}{i}$. 


\section{The Background Field Method for Noncommutative Gauge The- ories}

In this Section we discuss the set-up of the method for noncommutative $U(N)$ gauge theories.

We start off decomposing the gauge field $A_{\mu}$ into a background field $B_{\mu}$ and a fluctuating quantum field $N_{\mu}$,

$$
A_{\mu}=B_{\mu}+N_{\mu}
$$

$N_{\mu}$ is treated as a highly virtual field with momenta above the Wilsonian scale while the background field is taken to be slowly varying, but still fully noncommutative. We are interested in the Wilsonian effective action $S_{\text {Weff }}(B)$ which is obtained by functionally integrating over the fluctuating fields. For the pure gauge theory we have (schematically)

$$
\exp \left[-S_{\mathrm{Weff}}(B)\right]=\int \mathcal{D} N \exp \left[-S_{\mathrm{YM}}(A)\right]
$$

The noncommutative pure Yang-Mills action is given by (1.3). For a fixed background field the Yang-Mills action has a gauge symmetry for the fluctuating field $N_{\mu}$. Hence we need to fix the gauge and we will do this by adding to (1.3) the gauge-fixing functional [21]

$$
S_{\text {g.f. }}=-\frac{1}{g^{2}} \int d^{4} x \operatorname{Tr}\left(\left(D_{\mu}(B) N_{\nu}\right) \star\left(D_{\mu}(B) N_{\nu}\right)\right)
$$

together with the corresponding action for the ghost fields,

$$
S_{\text {ghost }}=-2 \int d^{4} x \operatorname{Tr}\left(\bar{c} \star D_{\mu}(B) \star D_{\mu}(B+N) \star c\right)
$$

Importantly, when the background gauge field $B_{\mu}$ is not held fixed, the gauge-fixed action $S_{\mathrm{YM}}+S_{\text {g.f. }}+S_{\text {ghost }}$ is gauge-invariant under gauge transformations of the background field $B_{\mu}$ and rotations of $N_{\mu}$ (and the ghosts), i.e.

$$
\begin{aligned}
B_{\mu} & \longrightarrow B_{\mu}^{\Omega}=\Omega\left(B_{\mu}+\partial_{\mu}\right) \Omega^{-1} \\
N_{\mu} & \longrightarrow N_{\mu}^{\Omega}=\Omega N_{\mu} \Omega^{-1} \\
c & \longrightarrow c^{\Omega}=\Omega c \Omega^{-1}
\end{aligned}
$$

where $\Omega(x)$ is an element of the noncommutative gauge group, $\Omega=e_{\star}^{\alpha^{A} t^{A}},[13]$.

In a one-loop computation of the effective action, one discards linear terms in the fluctuations and keeps track of the quadratic terms. After a little algebra we find that the gauge-fixed 
noncommutative Yang-Mills action functional can be rewritten as

$$
\begin{aligned}
S_{\mathrm{YM}}+S_{\text {g.f. }}+S_{\text {ghost }}= & -\frac{1}{2 g^{2}} \int d^{4} x \operatorname{Tr}\left(F_{\mu \nu}^{B} \star F_{\mu \nu}^{B}\right) \\
& -\frac{1}{g^{2}} \int d^{4} x \operatorname{Tr}\left(N_{\mu} \star\left[M_{\mu \nu}^{\text {g.f. }}, N_{\nu}\right]_{\star}\right)-2 \int d^{4} x \operatorname{Tr}\left(\bar{c} \star D^{2}(B) \star c\right)
\end{aligned}
$$

where

$$
M_{\mu \nu}^{\text {g.f. }}=-D^{2}(B) \delta_{\mu \nu}-2 F_{\mu \nu}^{B}
$$

In this derivation, we have repeatedly used the fact that $\int d^{4} x f_{1} \star f_{2} \star \cdots \star f_{n-1} \star f_{n}=\int d^{4} x f_{n} \star$ $f_{1} \star \cdots \star f_{n-1}$. Note that for any field $\phi$ transforming in the adjoint of the gauge group (as $N_{\mu}$ or c), i.e. $\phi^{\Omega}=\Omega \phi \Omega^{-1}$, the background covariant derivative is given by $D_{\mu}(B) \phi=\partial_{\mu} \phi+\left[B_{\mu}, \phi\right]_{\star}$, and

$$
D^{2}(B) \star \phi \equiv \partial^{2} \phi+\left[\left(\partial_{\mu} B_{\mu}\right), \phi\right]_{\star}+2\left[B_{\mu}, \partial_{\mu} \phi\right]_{\star}+\left[B_{\mu},\left[B_{\mu}, \phi\right]_{\star}\right]_{\star}
$$

After the functional integration over the $N$-fields (and the ghosts) in (2.2) we are left with an effective action of the $B$ fields only. Noncommutative gauge-invariance (2.5) is crucial in constraining the interactions which can be generated in this procedure. Consequently the Wilsonian effective action will always contain the 'kinetic term'

$$
S_{\mathrm{Weff}}[B] \ni-\frac{1}{2 g_{\text {eff }}^{2}} \int d^{4} x \operatorname{Tr}\left(F_{\mu \nu}^{B} \star F_{\mu \nu}^{B}\right)
$$

where the multiplicative coefficient on the right hand side is identified with the Wilsonian coupling constant at the corresponding momentum scale. Of course, in addition to this term there are higher-dimensional operators ${ }^{2}$, which are suppressed by inverse powers of the cutoff.

We can now generalize our analysis by adding to the pure gauge action generic matter fields: complex scalars and Weyl fermions transforming in general representations of the gauge group $^{3}$, which are described by the action

$$
S_{\text {fermi }}+S_{\text {scalar }}=-2 \int d^{4} x \operatorname{Tr}\left(\bar{\lambda} \bar{\sigma}_{\mu} \star\left(D_{\mu} \lambda\right)\right)-2 \int d^{4} x \operatorname{Tr}\left(\overline{\left(D_{\mu} \phi\right)} \star\left(D_{\mu} \phi\right)\right) .
$$

In what follows we will be interested in the term in the Wilsonian effective action quadratic in the background gauge field. For the purpose of determining the Wilsonian coupling constant

\footnotetext{
${ }^{2}$ It will soon turn out that in non-supersymmetric noncommutative theories the Wilsonian effective action also contains operators of lower dimension than $F_{\mu \nu}^{B} \star F_{\mu \nu}^{B}$. These are non-local operators, but perfectly consistent with noncommutative gauge invariance. These operators will be singular and unsuppressed in the infrared, leading to a rather abnormal behaviour of the theory [4].

${ }^{3}$ For a discussion of the allowed representations of the noncommutative $U(1)$ group see $[11,13-15]$.
} 
it is sufficient to concentrate on the kinetic term $\left(\partial_{\mu} B_{\nu}-\partial_{\nu} B_{\mu}\right)^{2}$ in the effective Lagrangian, which in momentum space gives $2 B_{\mu}(k) B_{\nu}(-k)\left(k^{2} \delta_{\mu \nu}-k_{\mu} k_{\nu}\right)$. In the effective theory the tree level transverse tensor $\left(k^{2} \delta_{\mu \nu}-k_{\mu} k_{\nu}\right)$ will in general be replaced by a tensor $\Pi_{\mu \nu}(k)$ which from now on will be referred to as the Wilsonian polarization tensor. It is defined so that the term in $S_{\text {Weff }}$ quadratic in the background gauge field is

$$
2 \int \frac{d^{4} k}{(2 \pi)^{4}} B_{\mu}(k) B_{\nu}(-k) \Pi_{\mu \nu} .
$$

In the effective theory arising from an underlying commutative theory, $\Pi_{\mu \nu}$ has the same tensor structure $f\left(k^{2}\right)\left(k^{2} \delta_{\mu \nu}-k_{\mu} k_{\nu}\right)$ as at the classical level due to Lorentz and gauge invariance. Only the scalar function $f\left(k^{2}\right)$ would receive contributions from perturbation theory. However, in noncommutative $\mathbb{R}^{4}$ there is another linearly-independent rank-2 symmetric transverse tensor: $\tilde{k}_{\mu} \tilde{k}_{\nu} / \tilde{k}^{4}$, where we have defined $\tilde{k}_{\mu}=\theta_{\mu \nu} k_{\nu}$. It is transverse, $k \cdot \tilde{k} \tilde{k}_{\nu} / \tilde{k}^{4}=0=\tilde{k}_{\mu} \tilde{k} \cdot k / \tilde{k}^{4}$, since $\theta_{\mu \nu}$ is antisymmetric. On general grounds, the gauge-boson polarization tensor has the structure

$$
\Pi_{\mu \nu}(k)=\Pi_{1}\left(k^{2}, \tilde{k}^{2}\right)\left(k^{2} \delta_{\mu \nu}-k_{\mu} k_{\nu}\right)+\Pi_{2}\left(k^{2}, \tilde{k}^{2}\right) \frac{\tilde{k}_{\mu} \tilde{k}_{\nu}}{\tilde{k}^{4}} .
$$

The new term $\tilde{k}_{\mu} \tilde{k}_{\nu} / \tilde{k}^{4}$ has the derivative dimension -2 , it is leading compared to the standard gauge-kinetic term (which has the derivative dimension +2 ), and singular in the infrared. We will show in Section 5 that $\Pi_{2}$ vanishes for all supersymmetric noncommutative gauge theories (unbroken and softly broken), as was first discussed in [4]. We will also see that $\Pi_{1}$ receives contribution from planar as well as from nonplanar diagrams, whereas $\Pi_{2}$ is an intrinsically noncommutative object and arises only from nonplanar diagrams.

One-loop computations in the background field method for a standard commutative theory literally are a textbook exercise [22]. To compute $\Pi_{\mu \nu}$ in noncommutative theories we will use the same elegant bookkeeping approach as in [22].

Let us introduce the action functional which describes the dynamics of a generic spin- $j$ noncommutative field in the representation $\mathbf{r}$ of the gauge group in the background of $B_{\mu}$ :

$$
\begin{aligned}
S[\phi] & =-\int d^{4} x \phi_{m, a} \star\left(-D^{2}(B) \delta_{m n} \delta^{a b}+2 i\left(F_{\mu \nu}^{B}\right)^{a b} \frac{1}{2} J_{m n}^{\mu \nu}\right) \star \phi_{n, b} \\
& \equiv-\int d^{4} x \phi_{m, a} \star\left[\Delta_{j, \mathbf{r}}\right]_{m n}^{a b} \star \phi_{n, b} .
\end{aligned}
$$

Here $a, b$ are indices of the representation $\mathbf{r}$ of the gauge group, $F^{a b} \equiv \sum_{A=1}^{N^{2}} F^{A} t_{a b}^{A}, m, n$ are spin indices and $J_{m n}^{\mu \nu}$ are the generators of the euclidean Lorentz group appropriate for the spin 
of $\phi$ :

$$
\begin{aligned}
J & =0 & & \text { for spin } 0 \text { fields } \\
J_{\rho \sigma}^{\mu \nu} & =i\left(\delta_{\rho}^{\mu} \delta_{\sigma}^{\nu}-\delta_{\rho}^{\nu} \delta_{\sigma}^{\mu}\right) & & \text { for } 4-\text { vectors } \\
{\left[J^{\mu \nu}\right]_{\alpha}^{\beta} } & =i \frac{1}{2}\left[\sigma^{\mu \nu}\right]_{\alpha}^{\beta} & & \text { for Weyl fermions } .
\end{aligned}
$$

Note that for fields transforming in the fundamental representation of the gauge group the appropriate covariant derivative is $D_{\mu}(B) \varphi=\left(\partial_{\mu}+B_{\mu}\right) \star \varphi$, so that $D^{2}(B) \varphi$ reads

$$
D^{2}(B) \star \varphi=\left(\partial^{2}+\left(\partial_{\mu} B_{\mu}\right)+2 B_{\mu} \partial_{\mu}+B_{\mu} \star B_{\mu}\right) \star \varphi .
$$

It then follows that, at one-loop level,

$$
S_{\mathrm{Weff}}[B]=-\frac{1}{2 g^{2}} \int d^{4} x \operatorname{Tr} F_{\mu \nu}^{B} \star F_{\mu \nu}^{B}-\sum_{j, \mathbf{r}} \alpha_{j} \log \operatorname{det}_{\star} \Delta_{j, \mathbf{r}},
$$

where the sum is extended to all fields in the theory, including ghosts and gauge fields. $\alpha_{j}$ is equal to +1 ( -1 ) for ghost (scalar) fields and to $+1 / 2(-1 / 2)$ for Weyl fermions (gauge fields). For the functional star-determinants we have

$$
\begin{aligned}
\log \operatorname{det}_{\star} \Delta_{j, \mathbf{r}} \equiv & \log \operatorname{det}_{\star}\left(-\partial^{2}+\mathcal{K}(B)_{j, \mathbf{r}}\right) \\
& =\log \operatorname{det}_{\star}\left(-\partial^{2}\right)+\operatorname{tr}_{\star} \log \left(1+\left(-\partial^{2}\right)^{-1} \mathcal{K}(B)_{j, \mathbf{r}}\right) .
\end{aligned}
$$

The first term on the second line of (2.19) will be dropped as it is $B$-independent and contributes only to the vacuum loops. The second term on the last line of (2.19) has an expansion in terms of Feynman diagrams. The next two sections are devoted to the computation of these diagrams.

\section{Feynman rules}

For simplicity from now on we will restrict our attention to the noncommutative $U(1)$ gauge group (modifications needed for the general $U(N)$ case will be considered elsewhere). To make our conventions similar to standard QED we change the normalization of the $U(1)$ generator to $t^{0}=1 / i$. We still use the anti-hermitean generator in order to keep $i$ 's out of the rest of the formulae.

\subsection{Adjoint representation}

Using (2.10) we rewrite $\Delta_{j, \mathbf{r}}$ acting on adjoint fields $\phi$ as

$$
\begin{aligned}
\Delta_{j, \mathrm{G}} \star \phi & \equiv-\partial^{2} \phi+\mathcal{K}(B)_{j, \mathrm{G}} \star \phi \\
& =-\partial^{2} \phi-\left[\left(\partial_{\mu} B_{\mu}\right), \phi\right]_{\star}-2\left[B_{\mu} \partial_{\mu}, \phi\right]_{\star}-\left[B_{\mu},\left[B_{\mu}, \phi\right]_{\star}\right]_{\star}+2 i\left(\frac{1}{2} J^{\mu \nu}\left[F_{\mu \nu}^{B}, \phi\right]_{\star}\right) .
\end{aligned}
$$


The Taylor expansion of the corresponding logarithm in (2.19) will involve the Feynman diagrams made from the three interaction vertices. The first one is the $\phi-B$ - $\phi$ vertex (which follows from the second and the third terms on the second line in (3.1)),

$$
\begin{aligned}
-\int d^{4} x \bar{\phi} \star\left[\left(\partial_{\mu} B_{\mu}\right)+2 B_{\mu} \partial_{\mu}, \phi\right]_{\star}= & \int \frac{d^{4} p^{\prime}}{(2 \pi)^{4}} \frac{d^{4} q}{(2 \pi)^{4}} \frac{d^{4} p}{(2 \pi)^{4}}(2 \pi)^{4} \delta^{(4)}\left(p+q-p^{\prime}\right) \\
& \bar{\phi}\left(p^{\prime}\right) B_{\mu}(q) \phi(p)\left[-2\left(p+p^{\prime}\right)_{\mu} \sin \frac{1}{2} q \tilde{p}\right] .
\end{aligned}
$$

The second vertex $\phi-B-B-\phi$ follows from the fourth term on the second line in (3.1),

$$
\begin{aligned}
-\int d^{4} x \bar{\phi} \star\left[B_{\mu},\left[B_{\mu}, \phi\right]_{\star}\right]_{\star}= & \int \frac{d^{4} p^{\prime}}{(2 \pi)^{4}} \frac{d^{4} q_{1}}{(2 \pi)^{4}} \frac{d^{4} q_{2}}{(2 \pi)^{4}} \frac{d^{4} p}{(2 \pi)^{4}}(2 \pi)^{4} \delta^{(4)}\left(p+q_{1}+q_{2}-p^{\prime}\right) \\
& \bar{\phi}\left(p^{\prime}\right) B_{\mu}\left(q_{1}\right) B_{\nu}\left(q_{2}\right) \phi(p)\left[-4 \delta_{\mu \nu} \sin \frac{1}{2} p^{\prime} \tilde{q}_{1} \sin \frac{1}{2} q_{2} \tilde{p}\right]
\end{aligned}
$$

and the third vertex follows from the last term on the second line in (3.1)

$$
\begin{aligned}
i \int d^{4} x \bar{\phi} J^{\mu \nu} \star\left[\partial_{\mu} B_{\nu}-\partial_{\nu} B_{\mu}, \phi\right]_{\star}= & \int \frac{d^{4} p^{\prime}}{(2 \pi)^{4}} \frac{d^{4} q}{(2 \pi)^{4}} \frac{d^{4} p}{(2 \pi)^{4}}(2 \pi)^{4} \delta^{(4)}\left(p+q-p^{\prime}\right) \\
& \bar{\phi}\left(p^{\prime}\right) J^{\mu \nu} B_{\nu}(q) \phi(p)\left[4 i q_{\mu} \sin \frac{1}{2} q \tilde{p}\right] .
\end{aligned}
$$

Notice that the interaction term arising from the commutator term in $F_{\mu \nu}^{B}$,

$$
\begin{aligned}
i \int d^{4} x \bar{\phi} J^{\mu \nu} \star\left[\left[B_{\mu}, B_{\nu}\right]_{\star}, \phi\right]_{\star}= & \int \frac{d^{4} p^{\prime}}{(2 \pi)^{4}} \frac{d^{4} q_{1}}{(2 \pi)^{4}} \frac{d^{4} q_{2}}{(2 \pi)^{4}} \frac{d^{4} p}{(2 \pi)^{4}}(2 \pi)^{4} \delta^{(4)}\left(p+q_{1}+q_{2}-p^{\prime}\right) \\
& \bar{\phi}\left(p^{\prime}\right) J^{\mu \nu} B_{\mu}\left(q_{1}\right) B_{\nu}\left(q_{2}\right) \phi(p)\left[4 i \sin \frac{1}{2} q_{1} \tilde{q_{2}} \sin \frac{1}{2} p \tilde{p^{\prime}}\right]
\end{aligned}
$$

does not contribute, to leading order, to the vacuum polarization tensor, since (3.5) vanishes when $q_{1}+q_{2}=0$.

The first two vertices (3.2) and (3.3) are the standard Feynman vertices for noncommutative electrodynamics with an adjoint scalar field and were first considered in [10]. The third expression (3.4) is the new, so-called $J$-vertex, specific to the background field method [22].

We now move on to fields in the fundamental representation.

\subsection{Fundamental representation}

We use (2.17) and rewrite $\Delta_{j, \mathbf{r}}$ acting on fundamental fields $\varphi$ as

$$
\left.\Delta_{j, \mathbf{f}} \star \varphi=\left\{-\partial^{2}-\left(\partial_{\mu} B_{\mu}\right)+2 B_{\mu} \partial_{\mu}+B_{\mu} \star B_{\mu}\right)+2 i\left(\frac{1}{2} J^{\mu \nu} F_{\mu \nu}^{B}\right)\right\} \star \varphi .
$$


The three vertices are then given by the following expressions:

$$
\begin{aligned}
& -\int d^{4} x \bar{\varphi} \star\left[\left(\partial_{\mu} B_{\mu}\right)+2 B_{\mu} \partial_{\mu}\right] \star \varphi=\int \frac{d^{4} p^{\prime}}{(2 \pi)^{4}} \frac{d^{4} q}{(2 \pi)^{4}} \frac{d^{4} p}{(2 \pi)^{4}}(2 \pi)^{4} \delta^{(4)}\left(p+q-p^{\prime}\right) \\
& \bar{\varphi}\left(p^{\prime}\right) B_{\mu}(q) \varphi(p)\left[-i\left(p+p^{\prime}\right)_{\mu} e^{-\frac{i}{2} q \tilde{p}}\right], \\
& -\int d^{4} x \bar{\varphi} \star\left(B_{\mu} \star B_{\mu}\right) \star \varphi=\int \frac{d^{4} p^{\prime}}{(2 \pi)^{4}} \frac{d^{4} q_{1}}{(2 \pi)^{4}} \frac{d^{4} q_{2}}{(2 \pi)^{4}} \frac{d^{4} p}{(2 \pi)^{4}}(2 \pi)^{4} \delta^{(4)}\left(p+q_{1}+q_{2}-p^{\prime}\right) \\
& \bar{\varphi}\left(p^{\prime}\right) B_{\mu}\left(q_{1}\right) B_{\nu}\left(q_{2}\right) \varphi(p)\left[-\delta_{\mu \nu} e^{-\frac{i}{2}\left(-p^{\prime} \tilde{q_{1}}+q_{2} \tilde{p}\right)}\right] \text {, } \\
& i \int d^{4} x \bar{\varphi} J^{\mu \nu} \star\left(\partial_{\mu} B_{\nu}-\partial_{\nu} B_{\mu}\right) \star \varphi=\int \frac{d^{4} p^{\prime}}{(2 \pi)^{4}} \frac{d^{4} q}{(2 \pi)^{4}} \frac{d^{4} p}{(2 \pi)^{4}}(2 \pi)^{4} \delta^{(4)}\left(p+q-p^{\prime}\right) \\
& \bar{\varphi}\left(p^{\prime}\right) J^{\mu \nu} B_{\nu}(q) \varphi(p)\left[-2 q_{\mu} e^{-\frac{i}{2} q \tilde{p}}\right] \text {. }
\end{aligned}
$$

Similar considerations to those presented before apply to the the term

$$
\begin{aligned}
i \int d^{4} x \bar{\varphi} J^{\mu \nu} \star\left[B_{\mu}, B_{\nu}\right]_{\star} \varphi= & \int \frac{d^{4} p^{\prime}}{(2 \pi)^{4}} \frac{d^{4} q_{1}}{(2 \pi)^{4}} \frac{d^{4} q_{2}}{(2 \pi)^{4}} \frac{d^{4} p}{(2 \pi)^{4}}(2 \pi)^{4} \delta^{(4)}\left(p+q_{1}+q_{2}-p^{\prime}\right) \\
& \bar{\varphi}\left(p^{\prime}\right) J^{\mu \nu} B_{\mu}\left(q_{1}\right) B_{\nu}\left(q_{2}\right) \varphi(p)\left[2 e^{-\frac{i}{2} p \tilde{p}^{\prime}} \sin \frac{1}{2} q_{1} \tilde{q_{2}}\right]
\end{aligned}
$$

which to leading order gives vanishing contribution to the vacuum polarization. Equations (3.7), (3.8) and (3.9) are the vertices for noncommutative electrodynamics with fundamental scalars, and the new vertex is specific to the background field treatment.

\section{Planar contributions}

Expanding the logarithm in (2.19) to the second order in the background fields $B_{\mu}$ gives the Feynman graphs shown in Figures 3,4 and 5 . We depict the $J$-vertices by a cross. The UV-divergent integrals will be regularized by using dimensional regularization, and the UVdivergences will be removed with the supersymmetry-preserving $\overline{\mathrm{DR}}$-scheme [23].

\subsection{Adjoint representation}

We start with the fields in the adjoint representation of noncommutative $U(1)$. 


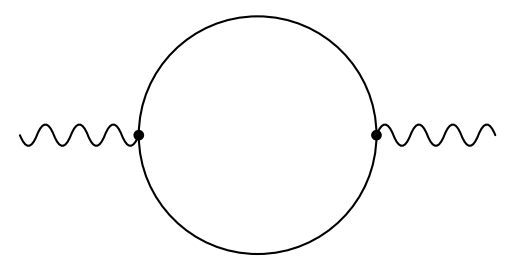

Figure 3.

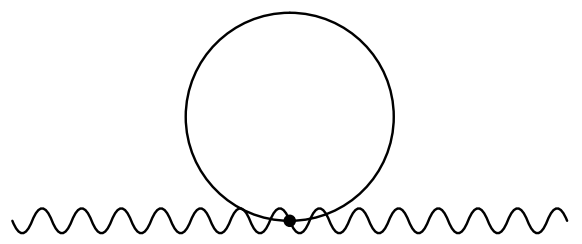

Figure 4.

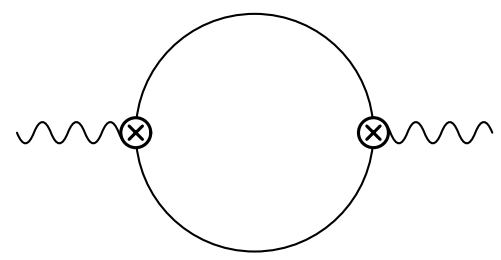

Figure 5. 
From the first Feynman amplitude (shown in Figure 3) we get

$$
-\frac{1}{2} \int \frac{d^{4} k}{(2 \pi)^{4}} B_{\mu}(k) B_{\nu}(-k) \int \frac{d^{D} p}{(2 \pi)^{D}} \operatorname{Tr} \frac{-4 \sin ^{2} \frac{1}{2} k \tilde{p}(2 p+k)_{\mu}(2 p+k)_{\nu}}{p^{2}(p+k)^{2}} .
$$

The second diagram, Figure 4, gives

$$
\int \frac{d^{4} k}{(2 \pi)^{4}} B_{\mu}(k) B_{\nu}(-k) \int \frac{d^{D} p}{(2 \pi)^{D}} \operatorname{Tr} \frac{-4 \sin ^{2} \frac{1}{2} k \tilde{p} \delta_{\mu \nu}}{p^{2}}
$$

where the trace is over spin indices. Its effect simply amounts to multiply the result by

$$
\operatorname{Tr} \mathbb{1}_{j} \equiv d(j)
$$

where $d(j)$ is the number of spin component of the field $\phi$,

$$
d(j) \equiv \quad 1 \text { for scalars, } \quad 2 \text { for Weyl fermions, } \quad 4 \text { for vectors. }
$$

We now examine the third amplitude. It is depicted in Figure 5 and gives

$$
-\frac{1}{2} \int \frac{d^{4} k}{(2 \pi)^{4}} B_{\mu}(k) B_{\nu}(-k) \int \frac{d^{D} p}{(2 \pi)^{D}} \operatorname{Tr} \frac{-16 J^{\mu \rho} J^{\nu \lambda} k_{\lambda} k_{\rho} \sin ^{2} \frac{1}{2} k \tilde{p}}{p^{2}(p+k)^{2}}
$$

where in the spin $j$ representation

$$
\begin{gathered}
\operatorname{Tr}\left(J^{\mu \rho} J^{\nu \lambda}\right)_{j}=C(j)\left(\delta^{\mu \nu} \delta^{\rho \lambda}-\delta^{\mu \lambda} \delta^{\nu \rho}\right) \\
C(j) \equiv \quad 0 \quad \text { for scalars, } \quad \frac{1}{2} \quad \text { for Weyl fermions, } \quad 2 \text { for vectors. }
\end{gathered}
$$

In what follows we will make an extensive use of identity

$$
\sin ^{2} \frac{1}{2} k \tilde{p}=\frac{1}{2}(1-\cos k \tilde{p}),
$$

and will refer to the contributions generated by the two terms on the right hand side of (4.8) as planar and non-planar contributions respectively.

Planar contributions to $\Pi_{\mu \nu}$ are selected with the substitution

$$
\sin ^{2} \frac{1}{2} k \tilde{p} \longrightarrow \frac{1}{2}
$$

into the sum of (4.1), (4.2) and (4.5), which we write as

$$
2 \int \frac{d^{4} k}{(2 \pi)^{4}} B_{\mu}(k) B_{\nu}(-k) \Pi_{\mu \nu}^{\text {planar }}(k ; j, \mathbf{G})
$$


where

$$
\begin{aligned}
\Pi_{\mu \nu}^{\text {planar }}(k ; j, \mathbf{G}) & =\frac{1}{2} \int \frac{d^{4} p}{(2 \pi)^{4}}\left\{\left[\frac{(2 p+k)_{\mu}(2 p+k)_{\nu}}{p^{2}(p+k)^{2}}-\frac{2 \delta_{\mu \nu}}{p^{2}}\right] C(\mathbf{G}) d(j)+4 \frac{k^{2} \delta_{\mu \nu}-k_{\mu} k_{\nu}}{p^{2}(p+k)^{2}} C(\mathbf{G}) C(j)\right\} \\
& =\left(\left[\Pi_{\mu \nu}^{\text {planar }}\right]_{1,2}+\left[\Pi_{\mu \nu}^{\text {planar }}\right]_{3}\right)(k ; j, \mathbf{G}) .
\end{aligned}
$$

Here $\left[\Pi_{\mu \nu}^{\text {planar }}\right]_{1,2}$ denotes the planar contributions arising from the sum of the first two Feynman diagrams, and $\left[\Pi_{\mu \nu}^{\text {planar }}\right]_{3}$ arises from the third one. Performing the $D$-dimensional integral, we get

$\left[\Pi_{\mu \nu}^{\text {planar }}\right]_{1,2}(k ; j, \mathbf{G})=\frac{1}{2}\left(k^{2} \delta_{\mu \nu}-k_{\mu} k_{\nu}\right)\left\{-\frac{d(j)}{(4 \pi)^{2}}\left[\frac{1}{3}\left(\frac{2}{\epsilon}-\gamma_{E}\right)-\int_{0}^{1} d x(1-2 x)^{2} \log \frac{A\left(k^{2}, x\right)}{4 \pi \mu^{2}}\right]\right\}$,

where $A\left(k^{2}, x\right)=k^{2} x(1-x)$, and we used $\Gamma\left(2-\frac{D}{2}\right)=\Gamma\left(\frac{\epsilon}{2}\right)=\frac{2}{\epsilon}-\gamma_{E}+O(\epsilon)$, where $\gamma_{E}$ is the Euler-Mascheroni constant. The mass parameter $\mu$ is introduced to keep the coupling constant dimensionless in $4-\epsilon$ dimensions, redefining $g \rightarrow g \mu^{\epsilon / 2}$.

We now compute the planar part of the third Feynman graph, which leads to

$$
\left[\Pi_{\mu \nu}^{\text {planar }}\right]_{3}(k ; j, \mathbf{G})=\frac{1}{2}\left(k^{2} \delta_{\mu \nu}-k_{\mu} k_{\nu}\right)\left\{\frac{4 C(j)}{(4 \pi)^{2}}\left[\frac{2}{\epsilon}-\gamma_{E}-\int_{0}^{1} d x \log \frac{A\left(k^{2}, x\right)}{4 \pi \mu^{2}}\right]\right\} .
$$

From (4.12) and (4.13) we extract the divergent part of the planar contribution,

$$
\Pi_{\mu \nu}^{d i v}(k ; j, \mathbf{G})=-\frac{1}{2}\left(k^{2} \delta_{\mu \nu}-k_{\mu} k_{\nu}\right)\left[\frac{\frac{d(j)}{3}-4 C(j)}{(4 \pi)^{2}}\right] \cdot\left(\frac{2}{\epsilon}\right)
$$

\subsection{Fundamental representation}

We now discuss the case of fundamental matter. It turns out that in this case the exponential factors mutually cancel in the first and third diagram, whereas become 1 in the second. In other words only the planar contribution is present ${ }^{4}$. More precisely, by comparing (3.2), (3.3), (3.4) to (3.7), (3.8), (3.9) respectively, one immediately realizes that the expressions for the Feynman graphs for fundamental matter are simply obtained from (4.1), (4.2), (4.5) by making the substitution

$$
4 \sin ^{2}(p \tilde{q} / 2) \longrightarrow 1
$$

Recalling (4.8), we conclude that the planar contribution from the adjoint field $\phi_{j}$ is exactly twice the one for matter fields in the fundamental, $\varphi_{j}$, whereas nonplanar contributions vanish.

\footnotetext{
${ }^{4}$ This was firstly seen in noncommutative electrodynamics in [12].
} 


\subsection{The microscopic $\beta$ function}

As usual, from (4.14) one can read the running of the renormalized coupling constant as a function of the subtraction point $\mu$, which in turn gives the $\beta$ function of the microscopic theory, $\beta(g)=\left[\mu \frac{\partial}{\partial \mu} g\right]_{g_{0}, \epsilon}$. This way we get

$$
\begin{aligned}
\beta(g) & =-\frac{b_{0}}{16 \pi^{2}} g^{3}+O\left(g^{5}\right), \\
b_{0} & =2 \sum_{j ; \mathbf{r}=\mathbf{f}, \mathbf{G}} \alpha_{j}\left[\frac{1}{3} d(j)-4 C(j)\right] C(\mathbf{r}),
\end{aligned}
$$

where we have formally defined

$$
C(\mathbf{G})=1, C(\mathbf{f})=\frac{1}{2}
$$

We stress that this $\beta$ function is defined in a mass-independent regularization scheme and does not change when massive particles are decoupled. The running of the Wilsonian coupling constant is correctly reproduced by it only in the extreme UV region.

The expression for $b_{0}$ in (4.16) can be recast in a more familiar form by noticing that the quantity $(1 / 3) d(j)-4 C(j)$ is equal to $1 / 3,-4 / 3,-20 / 3$ for complex scalars, Weyl fermions and gauge fields respectively. If we call $n_{\mathbf{r}_{\mathbf{f}}}\left(n_{\mathbf{r}_{\mathbf{b}}}\right)$ the number of Weyl fermions (complex scalars) transforming in the representation $\mathbf{r}_{\mathbf{f}}\left(\mathbf{r}_{\mathbf{b}}\right)$, we get, summing over all the fields in the theory,

$$
b_{0}=2\left(\frac{11}{3} C(\mathbf{G})-\frac{2}{3} \sum_{\mathbf{f}} n_{\mathbf{r}_{\mathbf{f}}} C\left(\mathbf{r}_{\mathbf{f}}\right)-\frac{1}{3} \sum_{\mathbf{b}} n_{\mathbf{r}_{\mathbf{b}}} C\left(\mathbf{r}_{\mathbf{b}}\right)\right) \text {. }
$$

The overall factor of 2 which multiplies (4.19) is merely a consequence of the choice of the $U(1)$ generator. Had we normalized it to $1 / 2$, as usually done for the $S U(N)$ generators in ordinary commutative Yang-Mills theories, this factor of 2 would not have been present.

\subsection{Supersymmetric theories}

Two observations are in order. First, notice that in supersymmetric theories there is a precise cancellation between bosonic and fermionic degrees of freedom,

$$
\sum_{j} \alpha_{j} d(j)=0, \text { for any } \mathbf{r} \text { of } G
$$

Second, let us explore in which theories the microscopic $\beta$ function vanishes. Using (4.20) and (4.17) we see that in this case

$$
\sum_{j, \mathbf{r}} \alpha_{j} C(\mathbf{r}) C(j)=0
$$


which means

$$
C(\mathbf{G})(4-\mathcal{N})=C(\mathbf{f}) N_{\mathbf{f}},
$$

where $\mathcal{N}$ is the number of supersymmetries and $N_{\mathbf{f}}$ the number of fundamental hypermultiplets. Therefore, pure $\mathcal{N}=4$ theories and $\mathcal{N}=2$ theories with $N_{\mathrm{f}}=2$ fundamental hypermultiplets have vanishing microscopic $\beta$ function.

Before closing this section, let us write down the full planar contribution to $\Pi_{\mu \nu}$ for a generic supersymmetric theory. First we define the scalar function $\Pi^{\text {planar }}\left(k^{2}\right)$ via

$$
\begin{aligned}
\Pi_{\mu \nu}^{\text {planar }}(k) & =\left(k^{2} \delta_{\mu \nu}-k_{\mu} k_{\nu}\right) \Pi^{\text {planar }}\left(k^{2}\right) \\
& \equiv \sum_{j, \mathbf{r}} \alpha_{j} \Pi_{\mu \nu}^{\text {planar }}(j, \mathbf{r})
\end{aligned}
$$

where the sum is over all fields in the theory. Then, using (4.20) and the definitions given in (4.18) we finally get

$$
\Pi^{\text {planar }}\left(k^{2}\right)=\frac{2}{(4 \pi)^{2}}\left(\sum_{j, \mathbf{r}} \alpha_{j} C(j) C(\mathbf{r})\right)\left[\frac{2}{\epsilon}-\gamma_{E}-\int_{0}^{1} d x \log \frac{A\left(k^{2}, x\right)}{4 \pi \mu^{2}}\right]+O(\epsilon) .
$$

We will make use of this expression in Section 6.

\section{Nonplanar contributions and IR/UV mixing}

As we have seen from the discussion preceeding (4.15), nonplanar contributions arise only from fields in the adjoint representation of noncommutative $U(1)$. They are read off from $(4.1),(4.2)$ and (4.5) with the substitution

$$
\sin ^{2} \frac{1}{2} k \tilde{p} \longrightarrow-\frac{1}{2} e^{\frac{i}{2} k \tilde{p}}
$$

These contributions are finite, so that we can work directly in $D=4$ dimensions and write

$$
2 \int \frac{d^{4} k}{(2 \pi)^{4}} B_{\mu}(k) B_{\nu}(-k) \Pi_{\mu \nu}^{n p}(k ; j, \mathbf{G})
$$

where

$$
\begin{aligned}
\Pi_{\mu \nu}^{n p}(k ; j, \mathbf{G})= & \frac{1}{2} \int \frac{d^{4} p}{(2 \pi)^{4}}\left(\left[\frac{-(2 p+k)_{\mu}(2 p+k)_{\nu}}{p^{2}(p+k)^{2}}+\frac{2 \delta_{\mu \nu}}{p^{2}}\right] C(\mathbf{G}) d(j)\right. \\
& \left.-4 \frac{k^{2} \delta_{\mu \nu}-k_{\mu} k_{\nu}}{p^{2}(p+k)^{2}} C(\mathbf{G}) C(j)\right) e^{i p \tilde{k}}
\end{aligned}
$$


Like in (2.14), let us decompose $\prod_{\mu \nu}^{n p}(k)$ as

$$
\Pi_{\mu \nu}^{n p}(k)=\Pi_{1}^{n p}\left(k^{2}, \tilde{k}^{2}\right)\left(k^{2} \delta_{\mu \nu}-k_{\mu} k_{\nu}\right)+\Pi_{2}^{n p}\left(k^{2}, \tilde{k}^{2}\right) \frac{\tilde{k}_{\mu} \tilde{k}_{\nu}}{\tilde{k}^{4}} .
$$

It is also convenient to introduce the quantities

$$
\hat{\Pi}=\delta_{\mu \nu}\left[\Pi^{n p}(k)\right]^{\mu \nu} \quad, \tilde{\Pi}=\frac{\tilde{k}_{\mu} \tilde{k}_{\nu}}{\tilde{k}^{2}}\left[\Pi^{n p}(k)\right]^{\mu \nu},
$$

which are related to $\Pi_{1}^{n p}, \Pi_{2}^{n p}$ via

$$
\Pi_{1}^{n p}=\frac{1}{2|k|^{2}}(\hat{\Pi}-\tilde{\Pi}) \quad, \Pi_{2}^{n p}=\frac{\tilde{k}^{2}}{2}(-\hat{\Pi}+3 \tilde{\Pi}) .
$$

Using

$$
\begin{aligned}
\int \frac{d^{4} p}{(2 \pi)^{4}} \frac{e^{i p \tilde{k}}}{p^{2}(p+k)^{2}} & =\frac{2}{(4 \pi)^{2}} \int_{0}^{1} d x K_{0}(\sqrt{A}|\tilde{k}|), \\
\int \frac{d^{4} p}{(2 \pi)^{4}} \frac{e^{i p \tilde{k}}}{p^{2}} & =\frac{1}{(4 \pi)^{2}} \frac{4}{\tilde{k}^{2}}
\end{aligned}
$$

where $A=k^{2} x(1-x)$, one finds

$$
\begin{aligned}
& \hat{\Pi}=\frac{C(\mathbf{G})}{(4 \pi)^{2}}\left\{\frac{8 d(j)}{\tilde{k}^{2}}-k^{2}[12 C(j)-d(j)] \int_{0}^{1} d x K_{0}(\sqrt{A}|\tilde{k}|)\right\}, \\
& \tilde{\Pi}=\frac{4 C(\mathbf{G})}{(4 \pi)^{2}}\left\{\frac{d(j)}{\tilde{k}^{2}}-\left(C(j) k^{2}-d(j) \frac{\tilde{k}_{\mu} \tilde{k}_{\nu}}{\tilde{k}^{2}} \frac{\partial}{\partial \tilde{k}_{\mu}} \frac{\partial}{\partial \tilde{k}_{\nu}}\right) \int_{0}^{1} d x K_{0}(\sqrt{A}|\tilde{k}|)\right\} .
\end{aligned}
$$

The Bessel function $K_{0}(z)$ has an expansion

$$
K_{0}(z)=-\log \frac{z}{2}\left[1+\frac{z^{2}}{4}+O\left(z^{4}\right)\right]-\gamma_{E}-\left(\gamma_{E}-1\right) \frac{z^{2}}{4}+O\left(z^{4}\right),
$$

which leads to the following expressions for $\Pi_{1}^{n p}, \Pi_{2}^{n p}$ :

$$
\begin{aligned}
& \Pi_{1}^{n p}\left(k^{2}, \tilde{k}^{2} ; j, \mathbf{G}\right) \simeq \\
& \quad-\frac{C(\mathbf{G})}{(4 \pi)^{2}}\left\{\left(\frac{d(j)}{3}-4 C(j)\right)\left(\log \frac{|k||\tilde{k}|}{2}+\gamma_{E}\right)+\frac{1}{2}\left[8 C(j)-\frac{5}{6} d(j)\right]\right\}, \\
& \Pi_{2}^{n p}\left(k^{2}, \tilde{k}^{2} ; j, \mathbf{G}\right) \simeq-\frac{C(\mathbf{G})}{(4 \pi)^{2}}\left\{-8 d(j)+\frac{d(j)}{4} k^{2} \tilde{k}^{2}\right\} .
\end{aligned}
$$

Notice that the $1 / \tilde{k}^{2}$ pole has exactly cancelled. We are now ready to sum over all fields in the theory, and find the complete nonplanar contribution to the vacuum polarization,

$$
\Pi_{\mu \nu}^{n p}=\sum_{j} \alpha_{j} \Pi_{\mu \nu}^{n p}(j, \mathbf{G})
$$


Recall from (5.4) that $\Pi_{2}$ is the coefficient in front of $\tilde{k}_{\mu} \tilde{k}_{\nu} / \tilde{k}^{4}$. This term has the same dimension as $\left(\partial_{\mu} B_{\nu}-\partial_{\nu} B_{\mu}\right)^{2}$, but it diverges quadratically as $\tilde{k} \rightarrow 0$. It gauge invariant, and it is not surprising that it has been generated.

In the case of supersymmetric theories, there is an important simplification, which follows from (4.20): the contributions arising from $\Pi_{2}$ sum up to zero, as first observed in [4]. Therefore, summing over all fields we get compact exact expressions

$$
\begin{aligned}
& \Pi_{1}^{n p}\left(k^{2}, \tilde{k}^{2}\right)=-\frac{4 C(\mathbf{G}) \sum_{j} \alpha_{j} C(j)}{(4 \pi)^{2}} \int_{0}^{1} d x K_{0}(\sqrt{A}|\tilde{k}|), \\
& \Pi_{2}^{n p}\left(k^{2}, \tilde{k}^{2}\right)=0 .
\end{aligned}
$$

Notice that a logarithmic singularity is generated in (5.12) whenever $k$ (or $\theta$ ) go to zero. However, this singularity is not present for $\mathcal{N}=4$ since in this case the sum over $j$ in the expression above is zero. These theories have vanishing $\beta$ function, and it is then natural to ask whether the logarithmic singularity also vanishes in $\mathcal{N}=2$ theories with two fundamental hypermultiplets, which have vanishing microscopic $\beta$ function as well. The answer to this question is no. In fact, no nonplanar contribution is generated by fundamental hypermultiplets circulating in loops, so the contribution coming from the $\mathcal{N}=2$ adjoint superfield is not cancelled.

Finally, note that for $\mathcal{N}=4$ theories (4.21) implies that both the planar and the nonplanar contribution to the vacuum polarization vanish:

$$
\left[\Pi_{\mu \nu}^{\text {planar }}\right]_{\mathcal{N}=4}=0,\left[\Pi_{\mu \nu}^{n p}\right]_{\mathcal{N}=4}=0 .
$$

\section{Flowing down from $\mathcal{N}=4$ and finite $\mathcal{N}=2$}

As an application of the Wilsonian approach to noncommutative gauge theories outlined in the previous sections, we now study how the decoupling of heavy degrees of freedom occurs in a noncommutative setup. In the previous analysis, we have found two examples of theories that are finite and free from quadratic infrared divergences: $\mathcal{N}=4$ pure supersymmetric YangMills and $\mathcal{N}=2$ supersymmetric Yang-Mills with two fundamental hypermultiplets. In the former, we can give mass to two of the four $(\mathcal{N}=1)$ chiral superfields, thus breaking $\mathcal{N}=4$ supersymmetry to $\mathcal{N}=2^{*}$. In the latter we give mass to the fundamental hypermultiplets. Naively the effective theories obtained in the two cases by integrating out massive degrees of freedom would be expected to belong to the same universality class in the extreme IR. To explore this issue we examine the behaviour of the Wilsonian coupling constants as functions of the momentum scale as this scale is lowered down from the UV-region to the infrared. 
We start with the $\mathcal{N}=2^{*}$ theory. Our previous construction outlined in Sections 4 and 5 is modified by the presence of non-zero mass $m$ for the two adjoint hypermultiplets. In general, the contribution to $\Pi_{\mu \nu}$ coming from a massive particle circulating in loops is taken into account by simply making in (4.24) and (5.15) the substitution

$$
A\left(k^{2}, x\right) \longrightarrow A\left(k^{2}, x ; m_{j, \mathbf{r}}^{2}\right) \equiv k^{2} x(1-x)+m_{j, \mathbf{r}}^{2}
$$

where $m_{j, \mathbf{r}}$ is the mass of a spin $j$ particle belonging to the representation $\mathbf{r}$ of the gauge group. This in turn implies that for $\mathcal{N}=2^{*}$ theories $\Pi_{\mu \nu}$ no longer vanishes; instead one has

$$
\begin{aligned}
\Pi_{1}^{n p} & =-\frac{4 C(\mathbf{G})}{(4 \pi)^{2}}\left(\sum_{j} C(j) \alpha_{j}\right) \int_{0}^{1} d x\left[K_{0}\left(\sqrt{k^{2} x(1-x)+m^{2}}|\tilde{k}|\right)-K_{0}\left(\sqrt{k^{2} x(1-x)}|\tilde{k}|\right)\right] \\
\Pi_{2}^{n p} & =0 \\
\Pi_{\text {planar }} & =-\frac{2 C(\mathbf{G})}{(4 \pi)^{2}}\left(\sum_{j} C(j) \alpha_{j}\right) \int_{0}^{1} d x \log \frac{m^{2}+k^{2} x(1-x)}{k^{2} x(1-x)}
\end{aligned}
$$

where the sum is over massive particles only. Therefore $\sum_{j} C(j) \alpha_{j} C(\mathbf{G})=(1 / 2) \cdot(1 / 2) \cdot 2=1 / 2$. Notice that, as anticipated in the Introduction, no IR-singularities appear in the nonplanar part, as in the massless case.

The Wilsonian coupling at the scale $k^{2}$ is given by

$$
\frac{1}{4 g_{\mathcal{N}=2^{*}}^{2}\left(k^{2}\right)}=\frac{1}{4 g_{\text {micro }}^{2}}+\Pi_{\mathcal{N}=2^{*}}\left(k^{2}\right)
$$

so that we conclude that

$$
\begin{aligned}
\frac{1}{g_{\mathcal{N}=2^{*}}^{2}\left(k^{2}\right)} & =\frac{1}{g_{\text {micro }}^{2}}+\frac{\left[b_{0}\right]_{\mathcal{N}=2^{\star}}}{(4 \pi)^{2}}\left\{\int_{0}^{1} d x \log \frac{k^{2} x(1-x)}{m^{2}+k^{2} x(1-x)}+\right. \\
& \left.+2 \int_{0}^{1} d x\left[K_{0}\left(\sqrt{k^{2} x(1-x)}|\tilde{k}|\right)-K_{0}\left(\sqrt{k^{2} x(1-x)+m^{2}}|\tilde{k}|\right)\right]\right\},
\end{aligned}
$$

where we have used $(4.19)$ to compute $\left[b_{0}\right]_{\mathcal{N}=2^{\star}}=2 \cdot(11 / 3-4 / 3-1 / 3)=4$. Note that the right hand side of (6.4) goes to $1 / g_{\text {micro }}^{2}$ as $k^{2} \rightarrow 0$. The running of the Wilsonian coupling can be conveniently described via

$$
\frac{1}{g_{\mathcal{N}=2^{*}}^{2}(k)}=\frac{1}{g_{\text {micro }}^{2}}+\frac{1}{2 \pi^{2}} f_{\mathcal{N}=2^{*}}\left(k, \theta^{-1 / 2}, m\right) .
$$

The function $f_{\mathcal{N}=2^{*}}\left(k, \theta^{-1 / 2}=10^{1}, m=10^{3}\right)$ is shown in Figure 6 . 


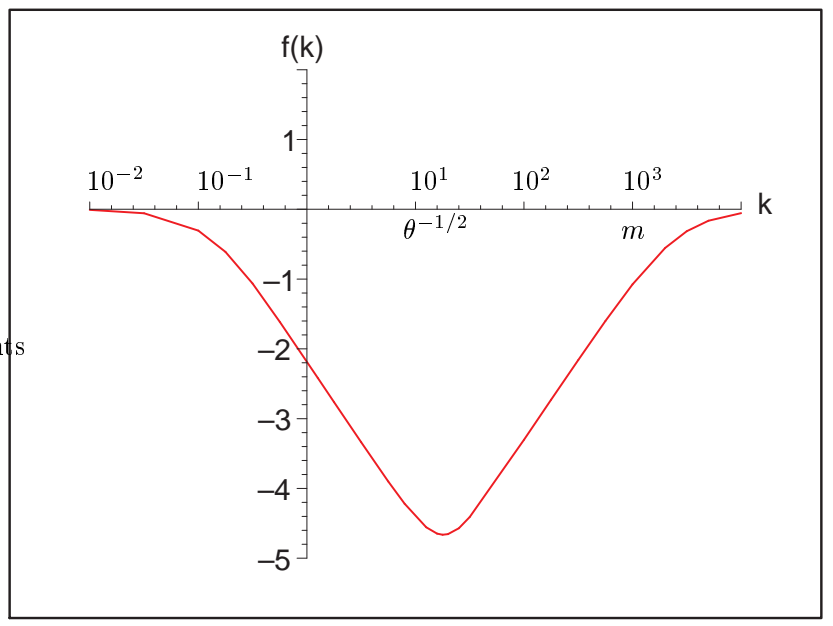

Figure 6. $\mathcal{N}=2^{*}$ running

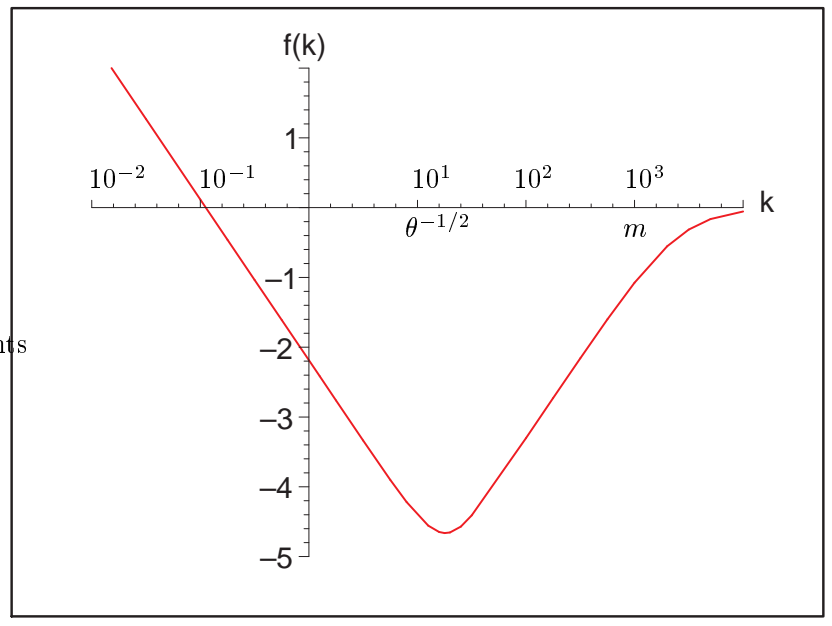

Figure 7. $\mathcal{N}=2$ running with 2 fundamental massive hypermultiplets 
Now we repeat the same analysis starting from $\mathcal{N}=2 U(1)$ supersymmetric Yang-Mills with two massive hypermultiplets and obtain

$$
\begin{aligned}
\Pi_{1}^{n p} & =\frac{2}{(4 \pi)^{2}} \int_{0}^{1} d x K_{0}\left(\sqrt{k^{2} x(1-x)}|\tilde{k}|\right), \\
\Pi_{2}^{n p} & =0 \\
\Pi_{\text {planar }} & =\frac{1}{(4 \pi)^{2}} \int_{0}^{1} d x \log \frac{k^{2} x(1-x)}{m^{2}+k^{2} x(1-x)} .
\end{aligned}
$$

The Wilsonian coupling at the scale $k^{2}$ is now given by

$$
\begin{aligned}
\frac{1}{g_{\mathcal{N}=2}^{2}\left(k^{2}\right)} & =\frac{1}{g_{\text {micro }}^{2}}+\frac{\left[b_{0}\right]_{\mathcal{N}=2}}{(4 \pi)^{2}}\left[\int_{0}^{1} d x \log \frac{k^{2} x(1-x)}{m^{2}+k^{2} x(1-x)}+\right. \\
& \left.+2 \int_{0}^{1} d x K_{0}\left(\sqrt{k^{2} x(1-x)}|\tilde{k}|\right)\right],
\end{aligned}
$$

where again, in the normalizations of (4.19), $\left[b_{0}\right]_{\mathcal{N}=2}=4$. Finally, we represent the running of the Wilsonian coupling via

$$
\frac{1}{g_{\mathcal{N}=2}^{2}(k)}=\frac{1}{g_{\text {micro }}^{2}}+\frac{1}{2 \pi^{2}} f_{\mathcal{N}=2}\left(k, \theta^{-1 / 2}, m\right)
$$

and plot the function $f_{\mathcal{N}=2}\left(k, \theta^{-1 / 2}=10, m=10^{3}\right)$ in Figure 7 . As anticipated earlier $f$ exhibits a logarithmic behaviour in the infrared.

\section{Wilsonian flow in asymptotically free supersymmetric theories}

Our general formalism can be instantly applied to asymptotically free supersymmetric gauge theories. In this Section we briefly analyze the evolution of the Wilsonian coupling constant in pure $\mathcal{N}=2$ and $\mathcal{N}=1$ Super Yang-Mills theories.

The Wilsonian coupling at the scale $k^{2}$ is given by

$$
\frac{1}{4 g_{\mathrm{eff}}^{2}\left(k^{2}\right)}=\frac{1}{4 g_{\mathrm{micro}}^{2}}+\Pi\left(k^{2}\right)
$$

where $\Pi=\Pi^{\text {planar }}+\Pi_{1}^{n p}$. The renormalized planar contribution $\Pi^{\text {planar }}$ is obtained from (4.24) by subtracting the pole in $\epsilon$ in the $\overline{\mathrm{DR}}$ scheme,

$$
\frac{1}{4 g_{\text {micro }}^{2}}+\Pi^{\text {planar }}\left(k^{2}\right)=-\frac{2}{(4 \pi)^{2}}\left(\sum_{j, \mathbf{r}} \alpha_{j} C(j) C(\mathbf{r})\right) \int_{0}^{1} d x \log \frac{k^{2} x(1-x)}{\Lambda_{\overline{\mathrm{DR}}}^{2}} .
$$


The nonplanar contribution was calculated in (5.15). Putting these results together, we obtain the running of the Wilsonian coupling,

$$
\frac{1}{g_{\mathrm{eff}}^{2}\left(k^{2}\right)}=\frac{b_{0}}{(4 \pi)^{2}}\left(\log \frac{k^{2}}{\Lambda_{\mathrm{DR}}^{2}}-2\right)+\frac{2 b_{0}}{(4 \pi)^{2}} \int_{0}^{1} d x K_{0}(\sqrt{x(1-x)}|k||\tilde{k}|),
$$

where $b_{0}$ is the first coefficient of the $\beta$ function given by (4.19). In our $U(1)$ normalization $\left[b_{0}\right]_{\mathcal{N}=2}=4$ and $\left[b_{0}\right]_{\mathcal{N}=1}=6$. From this we can easily read the UV and the IR limits of the $1 / g_{\mathrm{eff}}^{2}$ :

$$
\begin{aligned}
& \frac{1}{g_{\mathrm{eff}}^{2}} \rightarrow \frac{b_{0}}{(4 \pi)^{2}} \log k^{2}, \quad \text { as } k^{2} \rightarrow \infty, \\
& \frac{1}{g_{\mathrm{eff}}^{2}} \rightarrow-\frac{b_{0}}{(4 \pi)^{2}} \log k^{2}, \quad \text { as } k^{2} \rightarrow 0 .
\end{aligned}
$$

This shows that for not too large values of the microscopic coupling $g_{\text {micro }}$ the effective coupling

$g_{\text {eff }}$ does not diverge $\left(\frac{1}{g_{\text {eff }}^{2}}\right.$ does not reach zero). We saw that this was the case for the finite theories analyzed in the previous section. Remarkably this still holds in asymptotically free theories.

We conclude with the observation that standard holomorphy arguments [25] imply that the Wilsonian coupling in $\mathcal{N}=2$ theories is in fact one-loop exact in perturbation theory.

\section{Acknowledgements}

We would like to thank Nigel Glover, Gian Carlo Rossi and Massimo Testa for discussions. G.T. was supported by a PPARC SPG grant and the Angelo Della Riccia Foundation. 


\section{Appendix A: The NSVZ $\beta$ function for $U(N)$}

The $\beta$ function was computed for pure Yang-Mills theory with $U(1)$ group in $[7,8]$, for pure $U(N)$ in $[5,9,18]$ and for QED in [11] with ordinary perturbation theory. In Section 4.3 we have extended these results adding adjoint as well as fundamental matter for the gauge group $U(1)$. We now present an argument which leads to a generalization of the previous result (4.16) for supersymmetric $U(N)$ gauge theories. We will show that $b_{0}$ is still given by $(4.19)^{5}$ with the only replacement

$$
C(\mathbf{G})=N .
$$

The derivation is a simple extension of the well-known Novikov-Shifman-Vainshtein-Zakharov (NSVZ) derivation of the $\beta$ function in [26-28], so we will only sketch the argument. We limit our attention to pure supersymmetric gauge theories, and implicitly work with holomorphic gauge couplings (for recent discussions on the subtleties related to the definition of canonical and holomorphic gauge couplings and the presence of matter fields, we refer the reader to [29-31]) The first observation is that noncommutative gauge theories have instanton configurations, even in the case of gauge group $U(1)$ [32]. In the NSVZ derivation of the exact $\beta$ function, a key element was the renormalizability of the theory to all orders in perturbation theory. At present there is still no proof that supersymmetric theories are renormalizable, but strong evidence is supported by one-loop perturbative calculations; in [10] the one-loop renormalizability of $\mathcal{N}=2$ supersymmetric Yang-Mills theories on noncommutative on $\mathbb{R}^{4}$ was proven, while in $[16,17]$ it was shown that the Wess-Zumino model is renormalizable to all orders. We will then assume their renormalizability. In the calculation of instanton-dominated Green's functions, determinants of the kinetic operators of the quantum fluctuations around the instanton configuration cancel due to the d'Adda-Di Vecchia theorem [33], which is also at work in the noncommutative case. Then the result of a typical one-instanton amplitude is simply proportional to

$$
M^{n_{B}-\frac{1}{2} n_{F}} e^{-\frac{8 \pi^{2}}{g^{2}}} .
$$

Here $M$ is the Pauli-Villars mass scale which is usually introduced in supersymmetric instanton calculus, and $n_{B}\left(n_{F}\right)$ is the number of bosonic (fermionic) zero-modes of the Dirac operator on noncommutative $\mathbb{R}^{4}$ in the background of a charge one $U(N)$ instanton. In [32] it was shown that the moduli space of noncommutative $U(N)$ instantons is given by a resolution of singularities of the ordinary moduli space of commutative instantons (for recent reviews, see $[34,35])$. In the case at hand, we have precisely $n_{B}=4 N$. Supersymmetry relates this number to $n_{F}$, giving $n_{F}=\frac{1}{2} \mathcal{N} n_{B}$ where $\mathcal{N}$ is the number of supersymmetries; we conclude

\footnotetext{
${ }^{5}$ With the factor of 2 deleted, we return to the normalization $\operatorname{Tr}\left(t^{A} t^{B}\right)=-\delta^{A B} / 2, A, B=1, \ldots, N^{2}$
} 
that

$$
n_{B}-\frac{1}{2} n_{F}=4 N\left(1-\frac{\mathcal{N}}{4}\right)
$$

Under the stated assumptions, $M$ and $g=g(M)$ in (A.2) conspire to give a renormalization group invariant expression,

$$
M \frac{d}{d M}\left(M^{n_{B}-\frac{1}{2} n_{F}} e^{-\frac{8 \pi^{2}}{g^{2}(M)}}\right)=0
$$

from which it immediately follows that

$$
b_{0}[U(N)]=4 N\left(1-\frac{\mathcal{N}}{4}\right)
$$

Notice that (A.5) vanishes for $\mathcal{N}=4$, as in the commutative case.

\section{References}

[1] A. Connes, M.R. Douglas and A. Schwarz, JHEP 9802 (1998) 003, [hep-th/9711162].

[2] M.R. Douglas and C. Hull, JHEP 9802 (1998) 008, [hep-th/9711165].

[3] N. Seiberg and E. Witten, JHEP 9909 (1999) 032, [hep-th/9908142].

[4] A. Matusis, L. Susskind and N. Toumbas, "The IR/UV connection in the non-commutative gauge theories," hep-th/0002075.

[5] S. Minwalla, M. Van Raamsdonk and N. Seiberg, "Noncommutative perturbative dynamics," hep-th/9912072.

[6] T. Filk, "Divergences in a field theory on quantum space," Phys. Lett. B376 (1996) 53.

[7] M. M. Sheikh-Jabbari, "Renormalizability of the supersymmetric Yang-Mills theories on the noncommutative torus," JHEP 9906 (1999) 015 [hep-th/9903107].

[8] C. P. Martin and D. Sanchez-Ruiz, "The one-loop UV divergent structure of U(1) Yang-Mills theory on noncommutative $\mathbb{R}^{4}, "$ Phys. Rev. Lett. 83 (1999) 476 [hep-th/9903077].

[9] T. Krajewski and R. Wulkenhaar, "Perturbative quantum gauge fields on the noncommutative torus," Int. J. Mod. Phys. A15 (2000) 1011 [hep-th/9903187].

[10] I. Y. Arefeva, D. M. Belov, A. S. Koshelev and O. A. Rychkov, "Renormalizability and UV/IR mixing in noncommutative theories with scalar fields," Phys. Lett. B487 (2000) 357.

[11] M. Hayakawa, "Perturbative analysis on infrared aspects of noncommutative QED on $\mathbb{R}^{4}$," Phys. Lett. B478 (2000) 394 [hep-th/9912094]. 
[12] M. Hayakawa, "Perturbative analysis on infrared and ultraviolet aspects of noncommutative QED on $\mathbb{R}^{4}$, [hep-th/9912167].

[13] S. Ferrara and M. A. Lledo, "Some aspects of deformations of supersymmetric field theories," JHEP 0005 (2000) 008 [hep-th/0002084].

[14] S. Terashima, "A note on superfields and noncommutative geometry," Phys. Lett. B482 (2000) 276 [hep-th/0002119].

[15] J. M. Gracia-Bondia and C. P. Martin, "Chiral gauge anomalies on noncommutative $\mathbb{R}^{4}$," Phys. Lett. B479 (2000) 321 [hep-th/0002171].

[16] H. O. Girotti, M. Gomes, V. O. Rivelles and A. J. da Silva, "A consistent noncommutative field theory: The Wess-Zumino model," Nucl. Phys. B587 (2000) 299 [hep-th/0005272].

[17] A. A. Bichl, J. M. Grimstrup, H. Grosse, L. Popp, M. Schweda and R. Wulkenhaar, "The superfield formalism applied to the noncommutative Wess-Zumino model," JHEP 0010 (2000) 046 [hep-th/0007050].

[18] A. Armoni, "Comments on perturbative dynamics of non-commutative Yang-Mills theory," hep-th/0005208.

[19] J. Ambjorn, Y. M. Makeenko, J. Nishimura and R. J. Szabo, "Lattice gauge fields and discrete noncommutative Yang-Mills theory," JHEP 0005 (2000) 023 [hep-th/0004147].

[20] E. T. Akhmedov, P. DeBoer and G. W. Semenoff, "Running couplings and triviality of field theories on non-commutative spaces," hep-th/0010003.

[21] S. Weinberg, "Effective Gauge Theories," Phys. Lett. B91 (1980) 51.

[22] M. E. Peskin and D. V. Schroeder, "An Introduction to quantum field theory," Reading, USA: Addison-Wesley (1995).

[23] W. Siegel, "Supersymmetric Dimensional Regularization Via Dimensional Reduction," Phys. Lett. B84 (1979) 193.

[24] G. 't Hooft, "Computation of the quantum effects due to a four-dimensional pseudoparticle," Phys. Rev. D14 (1976) 3432.

[25] N. Seiberg, "Supersymmetry And Nonperturbative Beta Functions," Phys. Lett. B206 (1988) 75 .

[26] V. A. Novikov, M. A. Shifman, A. I. Vainshtein and V. I. Zakharov, "Exact Gell-Mann-Low Function Of Supersymmetric Yang-Mills Theories From Instanton Calculus," Nucl. Phys. B229 (1983) 381

[27] V. A. Novikov, M. A. Shifman, A. I. Vainshtein and V. I. Zakharov, "Instantons And Exact Gell-Mann-Low Function Of Supersymmetric O(3) Sigma Model," Phys. Lett. B139 (1984) 389. 
[28] V. A. Novikov, M. A. Shifman, A. I. Vainshtein and V. I. Zakharov, "Beta Function In Supersymmetric Gauge Theories: Instantons Versus Traditional Approach," Phys. Lett. B166 (1986) 329.

[29] M. Dine and Y. Shirman, "Some explorations in holomorphy," Phys. Rev. D50 (1994) 5389 [hep-th/9405155].

[30] N. Arkani-Hamed and H. Murayama, "Renormalization group invariance of exact results in supersymmetric gauge theories," Phys. Rev. D57 (1998) 6638 [hep-th/9705189].

[31] N. Arkani-Hamed and H. Murayama, "Holomorphy, rescaling anomalies and exact beta functions in supersymmetric gauge theories," JHEP 0006 (2000) 030 [hep-th/9707133].

[32] N. Nekrasov and A. Schwarz, "Instantons on noncommutative $\mathbb{R}^{4}$ and $(2,0)$ superconformal six dimensional theory," Commun. Math. Phys. 198, 689 (1998) [hep-th/9802068].

[33] A. D'Adda and P. Di Vecchia, "Supersymmetry and instantons," Phys. Lett. B73 (1978) 162.

[34] H. Nakajima, "Lectures on Hilbert Schemes of Points on Surfaces," Providence, Rhode Island: American Mathematical Society (1999).

[35] N. Dorey, T. J. Hollowood and V. V. Khoze, "A brief history of the stringy instanton", hep-th/0010015. 